\title{
Lung Infection, CTCAE
}

National Cancer Institute

\section{Source}

National Cancer Institute. Lung Infection, CT CAE. NCI Thesaurus. Code C143657.

A disorder characterized by an infectious process involving the lungs, including pneumonia. 